\title{
Erratum to: Writing quality predicts Chinese learning
}

\author{
Connie Qun Guan ${ }^{1}$ - Lindsay N. Harris ${ }^{2}$ • \\ Wanjin Meng ${ }^{3}$ - Charles A. Perfetti ${ }^{4}$
}

Published online: 4 April 2015

(C) Springer Science+Business Media Dordrecht 2015

\section{Erratum to: Read Writ \\ DOI 10.1007/s11145-015-9549-0}

The original article has been published incorrectly with an error in the affiliation. The correct version is given in this erratum.

The online version of the original article can be found under doi:10.1007/s11145-015-9549-0.

Connie Qun Guan

conniequnguan@gmail.com

$\bowtie$ Wanjin Meng

wanjinmeng@yahoo.com

1 University of Science and Technology Beijing, Beijing, China

2 Northern Illinois University, Dekalb, IL, USA

3 China National Institute of Education Sciences, Beijing, China

4 University of Pittsburgh, Pittsburgh, PA, USA 